\title{
Fetal Rescue in Fatal Evolution of a Pregnant Woman with COVID-19: A Case Report
}

\author{
H. Asmouki ${ }^{1 *}$, A. Jamal Eddine ${ }^{1}$, K. Elbehja ${ }^{1}$, A. Soumani ${ }^{1}$, A. Ziadi ${ }^{2}$, F. Douirek ${ }^{2}$, M. Rhezali ${ }^{2}$, Y. Zarouki ${ }^{2}$, AR. El
} $\mathrm{Adib}^{2}$, MA. Samkaoui ${ }^{2}$

\author{
${ }^{1}$ Department of Gynecology Obstetric, University Hospital Mohammed VI, Marrakech, Morocco \\ ${ }^{2}$ Department of Anesthesiology, Intensive Care and Emergency Medicine, University Hospital Mohammed VI, Marrakech, Morocco
}

DOI: $10.36347 /$ sjmcr.2021.v09i03.021

| Received: 20.02.2021 | Accepted: 11.03.2021 | Published: 18.03.2021

*Corresponding author: $\mathrm{H}$. Asmouki

Abstract

A pregnant woman (Gravida 5, Abortion 1, Gestational Age: 35 weeks) was referred to our tertiary referral hospital with complaints of asthenia, dry cough and dyspnea. She had pulse rate of 77 , respiratory rate of 50, with oral temperature of $38.5, \mathrm{O} 2$ sat: $83 \%$ in room air. Her chest CT on admission had bilateral patchy ground-glass features, nasopharyngeal swab for SARS-CoV-2 had a positive result. She was admitted with a diagnosis of COVID-19. And antepartum fetal monitoring was reassuring. During her hospitalization, O2 saturation progressively declined, which required a transfer to the ICU and acute respiratory care and support, leading to intubation and mechanical ventilation. With concerns for fetal well-being she underwent a cesarean delivery of a viable but premature neonate. With persistent postpartum ARDS ( $\mathrm{SaO} 220 \%-30 \%$ on maximal ventilator support) she experienced acute hypotension and bradycardia 3 hours later and died despite cardiopulmonary resuscitative efforts.

Keywords: Coronavirus disease in pregnancy, respiratory failure with COVID-19, preterm birth.

Copyright $\odot 2021$ The Author(s): This is an open-access article distributed under the terms of the Creative Commons Attribution 4.0 International License (CC BY-NC 4.0) which permits unrestricted use, distribution, and reproduction in any medium for non-commercial use provided the original author and source are credited.

\section{INTRODUCTION}

The first cases of COVID-19 were reported by officials in Wuhan, China, in December 2019 [1]. The disease quickly spread throughout the world and was declared a pandemic by the World Health Organization (WHO) on March 12, 2020.

The clinical course of COVID-19 in pregnant women is not totally understood and there is worry for increased disease in some pregnant women and an increased risk for spontaneous abortion, preterm birth, or morbidity or mortality in the fetus and the neonate $[2,3]$.

\section{Case Report}

A pregnant woman (Gravida 5, Abortion 1, Gestational Age: 35 weeks) was referred to Mohammed 6 Hospital, a tertiary university center in Marrakesh, Morocco with complaints of asthenia, dry cough and dyspnea. She had no significant past medical disease. She had pulse rate of 77 , respiratory rate of 50 , with temperature of $38.5, \mathrm{O} 2$ sat: $83 \%$ in room air and $94 \%$ with nasal flow $\mathrm{O} 2$. Obstetric ultrasound was performed and the fetuse was normal then she was transferred to the intensive care unit. Spiral lung and mediastinal CT scan without contrast injection was performed that showed diffuse bilateral ground-glass opacity and consolidation. Her SARS-CoV-2 test result was positive. She was initiated on hydroxychloroquine, Ceftriaxone, Moxifloxacin, azithromycin. 
H. Asmouki et al., Sch J Med Case Rep, Mar, 2021; 9(3): 274-276

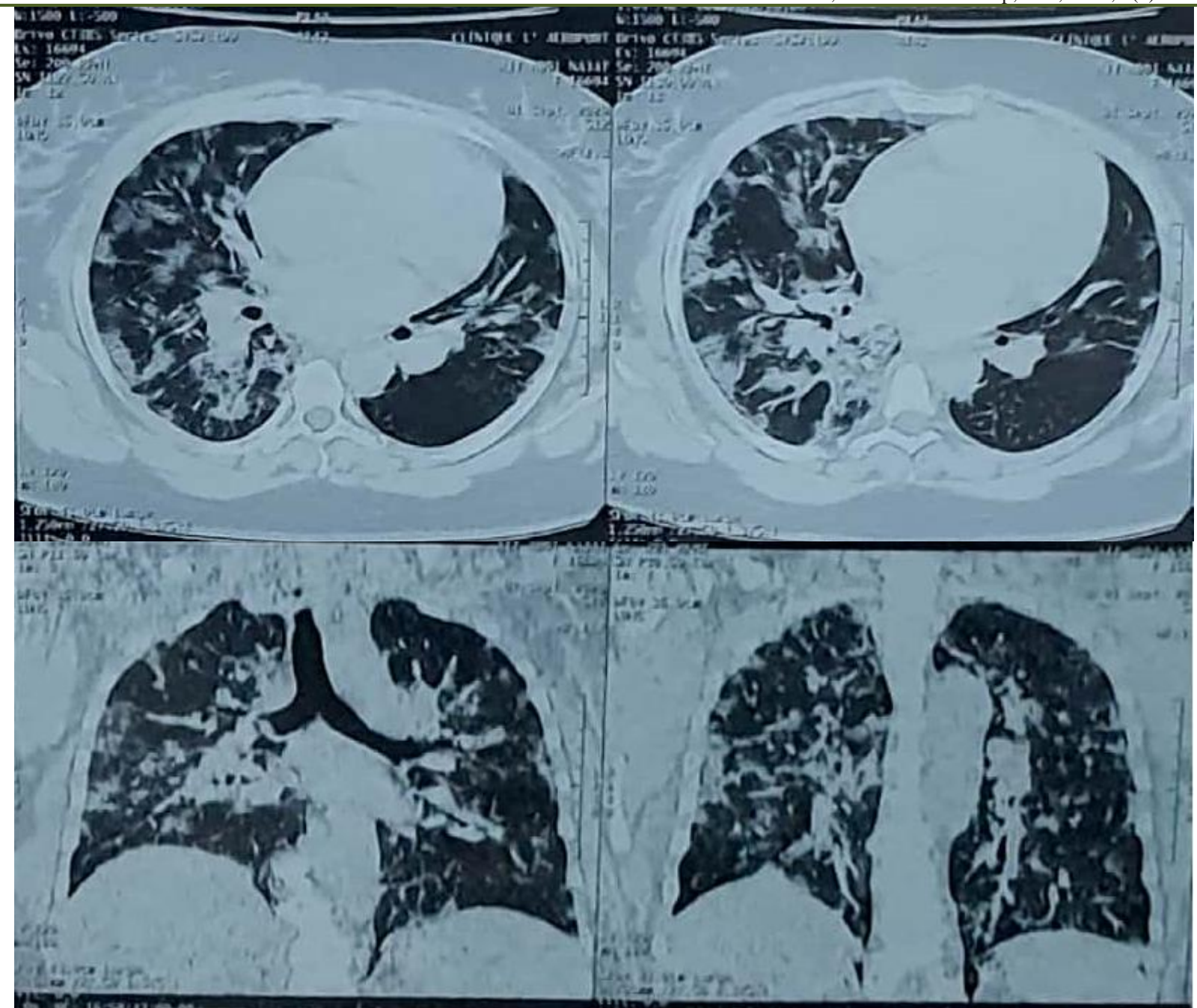

Figure: Chest CT scan: Multiple bilateral ground-glass opacities associated with pulmonary condensation predominantly peripherical and scattered at the level of the two lung fields, estimated at $75 \%$ of the lung volume

After 24 hours, the patient's condition worsened and $\mathrm{O} 2$ saturation dropped to $93 \%$, pulse rate of 107 , respiratory rate of 45 , supplemental $\mathrm{O} 2$ therapy with mask was needed (9L/min). After 72 hours in the ICU, due to progressive decline in respiratory status including increased respiratory rate to 58 breaths/min and decreased $\mathrm{O} 2$ saturation to $88 \%$, a noninvasive ventilation was required with $\mathrm{PEEP}=10 \mathrm{mmHg}$ and $\mathrm{FiO} 2=100 \%$. Then, the heart rate, respiratory rate and O2sat recovered to 123 beats/min, 40 breaths/min and $82 \%$, respectively. $48 \mathrm{~h}$ later, she acutely decompensated from ARDS with profound hypoxemia $(\mathrm{SaO} 2: 50 \%)$ she was intubated using fentanyl and propofol injection and mechanical ventilation was started. Despite her intubation, the patient's condition only worsened (SaO2:40\%). For severe COVID-19 with worsening respiratory status and concerns for fetal well-being, she underwent a cesarean delivery of viable but premature neonate. Even with maximal ventilator support, she experienced acute hypotension and bradycardia and died within 3 hours after failed resuscitative efforts.
Apgar of the newborn was $9 / 10$ changed to $10 / 10$ after $5 \mathrm{~min}$. the bith weight was $2800 \mathrm{~g}$. The neonate's pharyngeal swab was SARS-CoV-2 negative.

\section{DISCUSSION}

The recent COVID-19 pandemic has led to a unprecedented global health crisis. The speed with which the infection progressed, as well as the ambiguity of its impact on pregnancy due to the lack of scientific data, prompted obstetricians to adapt their practice based on pragmatic behavior [4].

Clinical manifestations (fever, cough, dyspnea, anosmia) are similar to those in non-pregnant patients.

Radiological explorations, in particular chest CT, should never be avoided because of pregnancy because the maternal vital prognosis can be involved in severe forms. Thus and to be practical, a careful assessment of the clinical condition, the need for hospitalization or even the indication of admission to an intensive care unit [5]. 
The management of patients with COVID-19 does not differ from the rest of the population apart from obstetric pathology. Symptomatic patients who present with respiratory failure, in particular, hospitalization in a COVID-19 department or even in an intensive care unit may be justified.

Concerning the molecules used, some teams use corticosteroid therapy whose impact on the progression of maternal disease is not available. However, all the learned societies unanimously recommend carrying out an antenatal pulmonary maturation before 34 weeks in the event of an unexpected delivery with an in utero transfer to a center adapted according to the gestational age, the estimated fetal weight and the pathologies maternal and / or fetal detected.

The need for preventive anticoagulant therapy is debated, but unpublished data report high rates of thromboembolic complications and open the question of routine prophylactic administration in any hospitalized pregnant patient with proven SARS-CoV-2 infection [6, 7].

Obstetrically, the route of delivery should not be influenced by the presence of SARS-CoV-2 infection, but guided by the usual obstetric indications and the clinical condition of the patient. Cesarean section may be indicated in patients with respiratory distress.

Obviously, special precautions must be taken within the nursing staff, in particular minimizing the number of people in contact with an infected patient and insisting on the wearing of protective equipment during childbirth, because of the risk of aerosolization.

\section{CONCLUSION}

We report the maternal death of a pregnant patient infected with SARS-CoV-2. Coronavirus disease 2019 during pregnancy, in the second or third trimester, is associated with an increased risk of maternal death and neonatal prematurity.

Understanding the impact of the COVID-19 pandemic on pregnancy experiences can help clarify clinical guidelines and protocols to help decrease maternal mortality and fetal prematurity rates.

\section{REFERENCES}

1. World Health Organization. Pneumonia of unknown caus: China. https://www.who.int/csr/don/05-january-2020pneumonia-of-unkown-cause-china/en/. Published January 5, 2020. Accessed June 6, 2020.

2. Di Mascio D, Khalil A, Saccone G, Rizzo G, Buca D, Liberati M, Vecchiet J, Nappi L, Scambia G, Berghella V, D'Antonio F. Outcome of coronavirus spectrum infections (SARS, MERS, COVID-19) during pregnancy: a systematic review and meta-analysis. American journal of obstetrics \& gynecology MFM. 2020 May $1 ; 2(2): 100107$.

3. Baud D, Greub G, Favre G, Gengler C, Jaton K, Dubruc E, Pomar L. Second-trimester miscarriage in a pregnant woman with SARS-CoV-2 infection. Jama. 2020 Jun 2;323(21):2198-200.

4. Chen H, Guo J, Wang C, Luo F, Yu X, Zhang W, Li J, Zhao D, Xu D, Gong Q, Liao J. Clinical characteristics and intrauterine vertical transmission potential of COVID-19 infection in nine pregnant women: a retrospective review of medical records. The lancet. 2020 Mar 7;395(10226):809-15.

5. Bowyer L, Robinson HL, Barrett H, Crozier TM, Giles M, Idel I. SOMANZ guidelines for the investigation and management sepsis in pregnancy. Aust N Z J Obstet Gynaecol. 2017 Oct;57(5):540-551.

6. Kimani J, Phiri K, Kamiza S, Duparc S, Ayoub A, Rojo R. Efficacy and safety of azithromycinchloroquine versus sulfadoxinepyrimethamine for intermittent preventive treatment of Plasmodium falciparum Malaria infection in pregnant women in Africa: an open-label, randomized trial. PLoS ONE. 2016 Jun 21;11(6): e0157045

7. Tookey PA, Thorne C, Wyk van J, Norton M. Maternal and fetal outcomes among 4118 women with HIV infection treated with lopinavir/ritonavir during pregnancy: analysis of population-based surveillance data from the national study of HIV in pregnancy and childhood in the United Kingdom and Ireland. BMC Infect Dis. 2016 Feb 4;16:65. 